\title{
Quality of Life Assessment with EQ-5D-3L in a Moroccan Diabetic Population
}

\author{
Fatima Zahra Kehailou' ${ }^{1}$, Mohammed Jabari' ${ }^{2}$, Ali Labriji ${ }^{3}$, Meryem Meftah El Khair ${ }^{1}$, \\ Hind Bouzoubaa ${ }^{1}$, Ghizlane Ouasmyne ${ }^{1}$, Oumaima El Moukhtari ${ }^{1}$, Souad El Amrani ${ }^{4}$, \\ Houriya Mestaghanmi ${ }^{1}$
}

\author{
${ }^{1}$ Department of Biology, Laboratory of Physiopathology and Molecular Genetics, Faculty of Sciences Ben M'sik, \\ University Hassan II-Casablanca, Casablanca, Morocco \\ ${ }^{2}$ General Direction of Services; Directorate of Sustainable Development and Urban Life, Prevention and Environmental Health \\ Division, Wilaya of the Casablanca-Settat Region, Ministry of the Interior, Casablanca, Morocco \\ ${ }^{3}$ Department of Computer Mathematics, Laboratory Modeling Analysis and Simulation, Faculty of Sciences Ben M'sik, University \\ Hassan II-Casablanca, Casablanca, Morocco \\ ${ }^{4}$ Department of Biology, Laboratory of Ecology and Environment, Faculty of Sciences Ben M'sik, University Hassan II-Casablanca, \\ Casablanca. Morocco \\ Email: fati.kehailou@gmail.com
}

How to cite this paper: Kehailou, F.Z., Jabari, M., Labriji, A., El Khair, M.M., Bouzoubaa, H., Ouasmyne, G., El Moukhtari, O., El Amrani, S. and Mestaghanmi, H. (2021) Quality of Life Assessment with EQ5D-3L in a Moroccan Diabetic Population. Open Access Library Journal, 8: e7357. https://doi.org/10.4236/oalib.1107357

Received: March 26, 2021

Accepted: May 23, 2021

Published: May 26, 2021

Copyright $\odot 2021$ by author(s) and Open Access Library Inc.

This work is licensed under the Creative Commons Attribution International License (CC BY 4.0).

http://creativecommons.org/licenses/by/4.0/

(c) (i) Open Access

\begin{abstract}
Diabetes is a chronic metabolic disease that impairs the lifestyle of patients in different physical, mental and social dimensions. This study aims to investigate the determinants of health-related quality of life (HRQoL) in diabetics. A cross-sectional study was conducted on 526 diabetic patients followed in 2019 at the Hygiene Department of the Sidi Othmane district (Casablanca). The data were collected using a questionnaire gathering the demographic, clinical and paraclinical information of patients, as well as a description of health status in the 5 dimensions by the EQ-5D questionnaire. The data was analyzed using SPSS version 23 software. The determinants of quality of life were assessed using the ANOVA test and the Pearson correlation test. The result is considered statistically significant at 0.05 . The average quality of life score based on the EQ-5D-3L scale and the VAS analog visual scale was $0.52 \pm 0.34$ and $55.47 \pm 15.50$, respectively. Severe problems were observed in anxiety/depression dimensions in $19.20 \%$ of patients, while the highest percentages for moderate problems were for the pain/physical discomfort and mobility dimensions $(53.80 \%$ and $36.90 \%$ respectively). The average scores of the EQ-5D-3L and visual analogue scale $(V A S)$ were significantly $(P<0.05)$ associated with age, gender, education, marital status, professional activity and complications. It follows from this study that the HRQoL of the population of diabetics studied is influenced by many factors, which must be considered in order to achieve better management of diabetes and improve their quality of life.
\end{abstract}




\section{Subject Areas}

Endocrinology

\section{Keywords}

Diabetes, Quality of Life, EQ-5D, EQ-VAS, Health Dimensions

\section{Introduction}

The rise of diabetes worldwide is the result of changes in style, lifestyle and an aging population. Diabetes is a major public health problem because of its increasing prevalence and incidence rates. According to the International Diabetes Federation (IDF) its prevalence was 463 million in 2019 and it will be in the order of 700 million by the year 2045 [1].

Diabetes is a chronic progressive disease. It is recognized as a major risk factor for cardiovascular disease, kidney disease and others. It is also a major cause of blindness, kidney failure, cardiovascular events, stroke and lower limb amputation [2]. It can affect the quality of life of patients through its physical but also psychological impact.

Health-related quality of life (HRQoL), it is the health status with three aspects physical, psychological and social, declared by the individuals themselves [3]. It is a way to assess how a person's well-being can be affected by a disease [3] [4]. Currently, it has become a major health issue. Its consideration allows the medical community to know how the patient experiences his disease [5].

The measure of HRQoL has the advantage of refocusing the patient care, of weighing the increasing technicality of medical procedures and of increasing the relevance of many medical decisions that are thus negotiated within the framework of a doctor-sick relationship [5] [6]. It is usually measured using questionnaires. Over the past few decades, the hundreds of HRQoL questionnaires have been developed to assess the quality of life of patients with many diseases [7] [8] [9]. In these questionnaires, we can distinguish between specific and generic instruments. Disease-specific instruments are more sensitive to detected changes in health status related to the disease, but due to of this specificity; comparisons between populations with different diseases are rarely possible [10]. On the other hand, generic instruments were built to assess the lifestyle of a general population without particularities or even to compare the lifestyle of groups of subjects to many pathologies [5] [11]. A number of generic measures have been developed and are being used, including Short Form-36 (SF-36), [12] [13] Short Form-12 (SF-12), [14] EQ-5D, [15] Nottingham Health Profile (NHP) [16] and Sickness Profile (SIP) [17].

Our study aims to assess lifestyle of a diabetic population using the generic EQ5D measurement instrument and to determine the factors that affect it. 


\section{Materials and Methods}

\subsection{Population Study}

This is a cross-sectional study of a population of 526 diabetic patients, followed by the Hygiene Department of the Sidi-Othman district, Casablanca-Morocco.

\subsection{Ethical Considerations}

This study was approved by the Biomedical Research Ethics Committees of Casablanca and Marrakech and was conducted in accordance with the principles of the Helsinki Declaration.

\subsection{Collect Data}

Diabetic patients were recruited from October 2019 to February 2020 at the Hygiene Department of the Sidi-Othman borough and diagnosed for at least a year. People under the age of 18 and pregnant women were excluded from this study.

A questionnaire was designed to collect information, including:

- Socio-demographic data (gender, age, marital status, education, professional activity).

- Clinical and medical data (type and age of diabetes, presence of complications, blood pressure...).

- Biological data (HbA1c, GAJ, GPP, blood pressure, cholesterol: CT, TG, HDL, LDL).

The blood glucose was measured by a One Call extra glucometer.

Blood pressure and pulse were measured using an Electronic Omron M6 Comfort blood pressure monitor.

Total cholesterol, LDL, HDL, triglycerides and HbA1c were dosed in laboratories of the private or public sectors of Casablanca.

For this study, we used the EQ-5D scale. It is a generic instrument that assesses the overall condition of patients (physical, psychological and social) regardless of their pathology [18]. The EQ-5D questionnaire consists in two parts:

- A descriptive part composed of five dimensions of health: mobility, self-care, usual activities, pain/discomfort and anxiety/depression.

- A vertical Visual Analogue Scale (EQ-VAS). The latter measuring $20 \mathrm{~cm}$ and graduated from 0 (the worst health condition) to 100 (the best health). It is a general assessment of the respondent's perceived health. It has four levels of answers: excellent, good, medium and bad. It is easy to use, just choose the level that well describes the patient's current state of health [19].

We preferred the EQ-5D 3L because we noticed that the answers for the EQ-5D 5L are very close. For each dimension of the EQ-5D 3L, the respondent has three options for answers: "no problems" rated 1, "moderate problems" rated 2, or "severe problems" rated 3. The answers given can be combined in a number of 5 digits, describing the respondent's state of health. The "11111" profile is a perfect health match designed for all 5 dimensions. The results of the 
EQ-5D dimensions can be transformed into a weighted index, ranging from -0.594 (poor health) to 1 (good health) [20].

The cross-cultural adaptation process of EQ-5D for Morocco was carried out according to the procedure indicated by the EuroQol International Group [15].

We have produced two translations of this scale from the French language to the Moroccan Arabic dialect by two teams of translators. Each is made up of two translators. The translations carried out by the two teams were compared, in the presence of investigators who had not been involved in the translation. The discrepancies between the two translated versions were discussed between the two teams, on the basis of which a final version adapted to Moroccan culture was developed.

The new translated version was against translated by two other different teams, mastering the French language and not having the original version of the EQ-5D scale. Two retro-translated versions of the questionnaire were developed, the synthesis of which was compared to the original French version.

The pre-final version of the questionnaire was pre-tested on a well-selected group of diabetic patients of different levels of knowledge, using both the descriptive EQ-5D (5-dimensional response) and EQ-VAS system. Each patient was asked about each question, to ensure that all the questions were properly understood by the respondents. Changes were made to the pre-final version and a final version of the questionnaire was developed.

The validated version of the EQ-5D scale was administered by the investigators to diabetics, after their information of the objectives of the study and the signature of informed consent.

\subsection{Statistical Analysis}

The SPSS version 23 software was used to analyze the collected data. The results were expressed in mean \pm standard deviation for all variables studied.

The calculation of the different health scores was made using a data sheet that we modeled on MS-Access, which allowed the automatic calculation of scores by dimension (scores between 0 and 100).

To identify the different factors affecting lifestyle, we studied the association between EQ-5D index and VAS scores and socio-demographic, socio-economic and clinical characteristics by the ANOVA (Analysis of variance) test for discontinuous variables and the Pearson test for continuous variables. The statistical significance has been set at $\mathrm{P}<00.05$.

The quota of the squares of factor and total deviations (partial eta-square (part eta squared)) has been calculated. It makes it easy to define the correlation ratio, also known as the non-linear correlation coefficient. It represents the proportion of variance of the dependent variable (the variable tested) explained by the independent variable (the group variable). This correlation ratio is always between 0 and 1 . The interpretation of this index was carried out according to Cohen (1988) [21]. 
Effet of Eta-square $\left(\eta^{2}\right)$ :

- Around $0.01=$ Small effect.

- Around $0.06=$ Medium effect.

- $\quad$ Around 0.14 and above $=$ Large effect.

\section{Results}

\subsection{Socio-Demographic and Socio-Economic Data}

This study involved 526 patients, of whom $75.9 \%$ are women and $24.1 \%$ are men. The average age was $54.56 \pm 12.86$ years. $61.2 \%$ of the population are married, $24 \%$ widowed, $10 \%$ single while only $4.8 \%$ are divorced. According to the level of education, $59.5 \%$ are illiterate, $20.5 \%$ have primary education and $16.9 \%$ have a secondary level (College: 10.6\%; High school: $6.3 \%$ ), while only $1.9 \%$ have completed a university course. In terms of professional activity, $86.7 \%$ of patients are unemployed (without occupation, housewives, retirees and students), compared to $13.3 \%$ who are employed. In terms of lifestyle, $96 \%$ of diabetics live in families, while $4 \%$ live alone. For medical coverage, 50.6\% benefited from the Medical Assistance Plan (RAMED), 24.5\% had the National Social Security Fund (CNSS); 6.8\% have the National Fund of Provident Organizations (CNOPS) and $18.1 \%$ of patients have no medical coverage (Table 1 ).

\subsection{Clinical, Biological and Lifestyle}

For the clinical characteristics of the population, $63.3 \%$ have type 2 diabetes (DNID) and 36.7\% type 1 diabetes (DID), with an average of diabetes duration $11.39 \pm 7.78$ years.

High blood pressure is present in $55 \%$ of diabetics. The most common chronic complication is retinopathy, followed by heart disease, neuropathy, arterial disease and kidney disease respectively $(25.6 \% ; 21.4 \% ; 16.10 \% ; 10.4 \% ; 6.4 \%)$. We also noticed that hyperglycemia is the most common acute complication in our diabetics $(35.8 \%)$ followed by hypoglycemia $(25.9 \%)$ coma $(9.4 \%)$, while the majority (98.90\%) have no complications (Table 2).

\subsection{EQ5D-3L Assesses the Quality of Life of Diabetics}

The average score for the EQ-5D index was $0.52 \pm 0.34$, ranging from -0.59 to 1 . While the health status scores of diabetics on the visual analog scale (VAS) was 55.47 vs. 15.50 and ranged from 25 to 100 .

Higher percentages of patients reported that they had no problems in different dimensions such as self-care (85.6\%), mobility (59.7\%) and usual activities (55.1\%). However, the highest percentages for moderate problems were reported in anxiety/depression (63.50\%) and in pain/discomfort (53.80\%). The highest percentages for extreme problems have been reported in some dimensions such as anxiety/depression (19.20\%), pain/discomfort and self-care with (11\%) (Table 3).

The EQ5D Score is strongly associated with physical activity $\left(P=0.0001 ; \eta^{2}=\right.$ 
Table 1. Socio-demographic and socio-economic characteristics of the diabetics surveyed.

\begin{tabular}{|c|c|}
\hline \multicolumn{2}{|c|}{ Variables } \\
\hline Age & \\
\hline Average age & $54.56 \pm 12.86$ years \\
\hline \multicolumn{2}{|l|}{ Gender } \\
\hline Men & $24.1 \%$ \\
\hline Women & $75.9 \%$ \\
\hline \multicolumn{2}{|c|}{ Professional activity } \\
\hline Yes & $13.30 \%$ \\
\hline Not & $86.70 \%$ \\
\hline \multicolumn{2}{|c|}{ Marital status } \\
\hline Single & $10 \%$ \\
\hline Married & $61.20 \%$ \\
\hline Divorced & $4.80 \%$ \\
\hline Widower & $24 \%$ \\
\hline \multicolumn{2}{|c|}{ Educational level } \\
\hline Illiterate & $59.50 \%$ \\
\hline Primary & $20.50 \%$ \\
\hline College & $10.60 \%$ \\
\hline Secondary & $6.30 \%$ \\
\hline University & $1.9 \%$ \\
\hline \multicolumn{2}{|l|}{ Lifestyle } \\
\hline Only & $4 \%$ \\
\hline Family & $96 \%$ \\
\hline \multicolumn{2}{|c|}{ Social coverage } \\
\hline RAMED & $50.60 \%$ \\
\hline Cnss & $24.50 \%$ \\
\hline Cnops & $6.80 \%$ \\
\hline No & $18.10 \%$ \\
\hline
\end{tabular}

$0.145)$ and chronic complications $\left(P=0.0001 ; \eta^{2}=0.092\right)$, which is moderately associated with the level of education $\left(P=0.0001 ; \eta^{2}=0.087\right)$ and marital status $\left(P=0.0001 ; \eta^{2}=0.055\right)$; while it is weakly associated with gender $\left(P=0.003 ; \eta^{2}=\right.$ 0.017); professional activity $\left(\mathrm{P}=0.011 ; \eta^{2}=0.012\right)$ and acute complications $(\mathrm{P}=$ $\left.0.003 ; \eta^{2}=0.017\right)$.

On the other hand, the VAS score shows a strong direct association with genre $\left(P=0.003 ; \eta^{2}=0.17\right)$, physical activity $\left(P=0.0001 ; \eta^{2}=0.104\right)$ and with the level of education $\left(P=0.0001 ; \eta^{2}=0.093\right)$. This score is moderately associated with marital status $\left(\mathrm{P}=0.0001 ; \eta^{2}=0.048\right)$ and chronic complications $\left(\mathrm{P}=0.0001 ; \eta^{2}=\right.$ 0.056) and weakly associated with marital status $\left(P=0.012 ; \eta^{2}=0.012\right)$ (Table 4). 
Table 2. Clinical characteristics and lifestyle of diabetics surveyed.

\begin{tabular}{|c|c|}
\hline \multicolumn{2}{|c|}{ Clinical variables } \\
\hline Diabetes duration & \\
\hline Average age & $11.39-7.78$ years \\
\hline \multicolumn{2}{|l|}{ Type of diabetes } \\
\hline T1D & $36.70 \%$ \\
\hline $\mathrm{T} 2 \mathrm{D}$ & $63.30 \%$ \\
\hline \multicolumn{2}{|l|}{ Smoking } \\
\hline Yes & $3.60 \%$ \\
\hline No & $96.40 \%$ \\
\hline \multicolumn{2}{|l|}{ Alcoholism } \\
\hline Yes & $0.80 \%$ \\
\hline No & $99.20 \%$ \\
\hline \multicolumn{2}{|l|}{ Physical activity } \\
\hline Yes & $37.50 \%$ \\
\hline No & $62.50 \%$ \\
\hline \multicolumn{2}{|l|}{ HBP } \\
\hline Yes & $55 \%$ \\
\hline No & $45 \%$ \\
\hline \multicolumn{2}{|c|}{ Chronic complications } \\
\hline Arteriopathy & $10.40 \%$ \\
\hline Heart & $21.40 \%$ \\
\hline Nephropathy & $6.40 \%$ \\
\hline Neuropathy & $16.10 \%$ \\
\hline Retinopathy & $25.60 \%$ \\
\hline Other complications & $7.90 \%$ \\
\hline No Complications & $12.80 \%$ \\
\hline \multicolumn{2}{|l|}{ Acute complications } \\
\hline Hyperglycemia & $35.80 \%$ \\
\hline Hypoglycemia & $25.90 \%$ \\
\hline Coma & $9.40 \%$ \\
\hline No complications & $28.90 \%$ \\
\hline
\end{tabular}

The study of correlations generally shows an inverse correlation between the scores of EQ-5D and VAS respectively, age $\left(\mathrm{P}=0.0001 ; \mathrm{r}^{2}=-0.273\right) ;(\mathrm{P}=$ $\left.0.0001 ; r^{2}=-0.242\right)$, the duration of diabetes $\left(P=0.0001 ; r^{2}=-0.164\right) ;(P=$ $\left.0.011 ; \mathrm{r}^{2}=-0.111\right)$ and blood pressure $\left(\mathrm{p}=0.0001 ; \mathrm{r}^{2}=-0.186\right) ;\left(\mathrm{P}=0.007 ; \mathrm{r}^{2}=\right.$ -0.144) (Table 5). This shows that most of these parameters increase, the patient's quality of life deteriorates.

\section{Discussion}

In this study, which aimed to assess the lifestyle of a population of diabetics 
Table 3. Patient health description for each quality of life level.

\begin{tabular}{lc}
\hline \multicolumn{1}{c}{ Dimensions EQ5D } & Percentage (\%) \\
\hline Mobility & $59.30 \%$ \\
No problem & $36.90 \%$ \\
Moderate problem & $3.80 \%$ \\
Extreme problem & \\
Self-care & \\
No problem & $85.60 \%$ \\
Moderate problem & $9.50 \%$ \\
Extreme problem & $4.90 \%$ \\
Usual activities & \\
No problem & $55.10 \%$ \\
Moderate problem & $33.30 \%$ \\
Extreme problem & $11.60 \%$ \\
Pain/discomfort & \\
No problem & \\
Moderate problem & $35.20 \%$ \\
Extreme problem & $53.80 \%$ \\
Anxiety/Depression & $11 \%$ \\
No problem & \\
Moderate problem & $17.30 \%$ \\
Extreme problem & \\
\hline & \\
\hline
\end{tabular}

followed by the Hygiene Department of the Sidi Othmane district (Casablanca, MAROC) using the EQ-5D-3L questionnaire, the average score for the lifestyle and VAS scale was 0.52 and 55.47, respectively. This is consistent with the results of Lyhyaoui who obsessed in type 2 diabetics that VAS was 56.66 [22]. Diabetes appears to be a health-degrading and health-altering condition of patients with diabetes. This alteration in quality of life varies according to studies. Thus, Cardoso observed in Portugal, that the average score of the EQ-5D was 0.67 and the VAS score was $64.85,[23]$ while Abedini who conducted a study in Iran, the score of the EQ-5D was 0.89 and VAS was 65.22 [24]. This difference in the lifestyle of diabetics could be explained by the actions of some factors (socio-economic, clinical and paraclinical...) that could affect it. Thus, these indicators must be taken into account when evaluating the results of the studies.

We observed that the majority of patients had no problems or reported mild problems in all dimensions especially, mobility and self-care, while moderate and severe problems were more common in the dimensions of anxiety/depression, usual activities and pain/discomfort. These results are consistent with many studies that have reported that the pain and depression were the main complaints of patients [25] [26]. On the other hand, Lyhyaoui, in Fez in 2011, also reported that the most extreme diabetic complaints were predominant, especially in the dimensions of pain and depression [22]. Along with 
Table 4. Association between average quality of life score VAS scale and qualitative variables.

\begin{tabular}{|c|c|c|c|c|c|c|c|}
\hline \multirow{2}{*}{ Variables } & & \multicolumn{3}{|c|}{ Score EQ-5D-3L } & \multicolumn{3}{|c|}{ Score VAS } \\
\hline & & Mean $+S d$ & ANOVA & $n^{2}$ & Avernge FT & Mean + Sd & $n^{2}$ \\
\hline \multirow{2}{*}{ Gender } & Men & $0.60 \pm 0.35$ & \multirow{2}{*}{0.003} & \multirow{2}{*}{0.017} & $59.06 \pm 16.86$ & \multirow{2}{*}{0.003} & \multirow{2}{*}{0.17} \\
\hline & Women & $0.50 \pm 0.34$ & & & $54.32 \pm 14.88$ & & \\
\hline \multirow{2}{*}{ Type of diabetes } & DT1 & $0.49 \pm 0.36$ & \multirow{2}{*}{0.084} & \multirow{2}{*}{0.006} & $54.40 \pm 16.73$ & \multirow{2}{*}{0.232} & \multirow{2}{*}{0.003} \\
\hline & DT2 & $0.54 \pm 0.33$ & & & $56.08 \pm 14.73$ & & \\
\hline \multirow{2}{*}{ Professional activity } & With profession & $0.63 \pm 0.32$ & \multirow{2}{*}{0.011} & \multirow{2}{*}{0.012} & $58.61 \pm 18.75$ & \multirow{2}{*}{0.092} & \multirow{2}{*}{0.005} \\
\hline & No profession & $0.51 \pm 0.34$ & & & $55.05 \pm 14.99$ & & \\
\hline \multirow{2}{*}{ Alcoholism } & Yes & $0.76 \pm 0.20$ & \multirow{2}{*}{0.174} & \multirow{2}{*}{0.004} & $55.41 \pm 15.51$ & \multirow{2}{*}{0.363} & \multirow{2}{*}{0.002} \\
\hline & Not & $0.52 \pm 0.34$ & & & $62.50 \pm 14.43$ & & \\
\hline \multirow{2}{*}{ Smoking } & Yes & $0.63 \pm 0.40$ & \multirow{2}{*}{0.176} & \multirow{2}{*}{0.03} & $59.21 \pm 14.93$ & \multirow{2}{*}{0.284} & \multirow{2}{*}{0.002} \\
\hline & Not & $0.52 \pm 0.34$ & & & $55.33 \pm 15.51$ & & \\
\hline \multirow{3}{*}{ Physical activity } & Yes & $0.69 \pm 0.23$ & \multirow{2}{*}{0.0001} & \multirow{2}{*}{0.145} & $61.93 \pm 15.27$ & \multirow{2}{*}{0.0001} & 0100 \\
\hline & Not & $0.42 \pm 0.36$ & & & $51.60 \pm 14.32$ & & 0.104 \\
\hline & Single & $0.64 \pm 0.30$ & & & $63.21 \pm 15.96$ & & \\
\hline M .1 - 1 & Married & $0.56 \pm 0.31$ & & onor & $56.13 \pm 14.74$ & $0000 \mathrm{O}$ & \\
\hline Heartal status & Divorced & $0.46 \pm 0.31$ & 0.0001 & 0.053 & $53 \pm 19.52$ & 0.0001 & 0.040 \\
\hline & Widower & $0.39 \pm 0.40$ & & & $50.99 \pm 14.96$ & & \\
\hline & Illiterate & $0.45 \pm 0.36$ & & & $52.27 \pm 14.59$ & & \\
\hline & Primary & $0.57 \pm 0.27$ & & & $56.94 \pm 14.43$ & & \\
\hline Educational level & College & $0.72 \pm 0.29$ & 0.0001 & 0.087 & $63.84 \pm 15.75$ & 0.0001 & 0.093 \\
\hline & Secondary & $0.68 \pm 0.28$ & & & $62.12 \pm 16.67$ & & \\
\hline & University & $0.76 \pm 0.13$ & & & $72.50 \pm 14.19$ & & \\
\hline & Yes & $0.46 \pm 0.35$ & & & $53.46 \pm 62.19$ & & \\
\hline Cironic compincalions & Not & $0.71 \pm 0.24$ & 0.0001 & 0.092 & $62.19 \pm 16.49$ & 0.0001 & 0.050 \\
\hline & Yes & $0.49 \pm 0.34$ & $0000+8$ & & $54.30 \pm 14.82$ & $0_{0} 010$ & \\
\hline Acule compincations & Not & $0.59 \pm 0.35$ & 0.003 & 0.017 & $57.89 \pm 16.59$ & 0.012 & 0.012 \\
\hline
\end{tabular}

Table 5. Correlation between average quality of life score, VAS scale and quantitative variables.

\begin{tabular}{ccccc}
\hline \multirow{2}{*}{ Variables } & \multicolumn{2}{c}{ Score EQ-5D } & \multicolumn{2}{c}{ Score VAS } \\
\cline { 2 - 5 } & $\mathrm{p}$-Value & Correlation coefficient $\left(\mathrm{r}^{2}\right)$ & $\mathrm{p}$-Value & Correlation coefficient $\left(\mathrm{r}^{2}\right)$ \\
\hline Age & 0,0001 & -0.273 & 0.0001 & -0.242 \\
Diabetes elderly & 0.0001 & -0.164 & 0.011 & -0.111 \\
HbA1 & 0.496 & 0.034 & 0.516 & 0.032 \\
Blood pressure & 0.0001 & -0.186 & 0.007 & -0.144 \\
\hline
\end{tabular}

different work in this field, our study also confirmed that most patients complained of moderate to severe problems with depression, pain and every day activities [24].

Our results revealed a significantly higher quality of life in men compared to 
women $(P=0.003)$, similar results were obtained with the VAS scale $(P=0.003)$. This finding is consistent with that of Abedini who also found that average scores for men's quality of life $(0.92 \pm 0.12)$ were significantly higher $(\mathrm{P}=0.004)$ than those of women $(0.86 \pm 0.13)$, for the VAS scale, the scores were $68.85 \pm$ 8.20 and $62.73 \pm 9.25$ respectively $(\mathrm{P}=0.008)$ [24]. This could be explained by the fact that women have shown a higher tendency to express health-related problems compared to men, thus lowering the quality of life score. This result agrees with that of Quah who showed that women had a significantly lower score of EQ-5D than men $(0.82 \pm 0.21 ; 0.89 \pm 0.17 ; \mathrm{P}<0.0001)$. On the other hand, they observed that the score of the VAS scale in women was lower than that of men, without any statistically significant difference $(68.4 \pm 16.8 ; 70.2 \pm$ 16.4; $\mathrm{P}<0.151$ ) [27].

Our results showed an inverse association between EQ5D, age $(p=0.0001)$ and duration of diabetes $(\mathrm{p}=0.0001)$, suggesting that HRQoL decrease with age. Similar results were observed by Abedini in Iran, which have shown that the HRQoL of diabetic patients decreases with age and with the duration of diabetes $(\mathrm{P}<0.05)$ [24]. The influence of age on quality of life may be due to a direct effect of aging and indirectly by the effect of aging on the dimensions that influence HRQoL [28]. On the other hand, O'Reilly have shown that quality of life scores increase with age, [29] which could be due to different economic and social conditions in many societies, [24] while other studies have found that more the duration of diabetes greater, more the HRQoL decreases [30].

In the present study, we observed that a lower HRQoL was related to widowed or divorced patients compared to married or single people with a higher HRQoL. We also found a significant correlation [24] between marital status and quality of life $(P=0.0001)$. Indeed, intimate attachment to other human beings is the pivot around which a person's life revolves, from birth to old age. Thus, the fact of being married helps draw strength and joy of life of the other, while the divorce reports a sharp drop in life satisfaction and well-being. [31] Our results are consistent with other authors reported that those separated/divorced/ widowed were more likely to have lower HRQoL than others [32] [33]. This suggests that marital status could be a good predictor of health.

In this study, diabetics with a professional activity had a better HRQoL $(0.63 \pm$ $0.32)$ compared to diabetics who did not $(0.51 \pm 0.34)(\mathrm{P}=0.011)$. Our result is consistent with that [31] of some authors [34] [35] [36]. This could be explained by having a job and a medium to high income can give a life satisfaction, because the subjects are more likely to access health services [37]. This was already demonstrated by Saleh who observed that income plays an important role in HRQoL [38].

In addition, higher-educated diabetics were more likely to have a good HRQoL compared to those with lower levels of education. Patients with a good level of education have positive self-esteem, better knowledge and a better understanding of the disease, its treatment and its complications [39]. Similar re- 
sults were observed by several authors [40].

Our results also suggest that the average quality of life score for diabetics engaged in physical activity is significantly higher $(\mathrm{P}=0.0001)$ than that of non-practicing people. A similar result was observed by Cardosso and his collaborators who noted that people engaged in physical activity have a higher score in EQ-5D and EQ-VAS and therefore, a better HRQoL [23].

In our study, we not only observed a relationship of complications of diabetes with a deterioration in quality of life, but also that high levels of $\mathrm{HbAlc}$ are associated with a decrease in the QOL score. This result perfectly matches those of Abdeni who showed that patients with an HbAlc level below 7 had a higher HRQoL score than those with a level above 7 [24]. Furthermore, the results of Solli show that people suffering from complications related to diabetes had a negative impact on their HRQoL [41]. Given the direct correlation between the complications of diabetes and good glycemic control [41] and the fact that the HbA1c level is indicative of glycemic status in the past 3 months, patients with low HbAlc levels should have a better HRQoL and fewer complications [42].

\section{Conclusion}

The results of this study emphasize the impact of diabetes on quality of life. It also appears that advanced age, female sex, low level of education, marital status (widowed or divorced), the presence of complications, were significantly associated with a decrease in the level of HRQoL and VAS scale. Therefore, improving the HRQoL of diabetics requires appropriate care, as well as an improvement in the socio-economic level and the level of education of the patients.

\section{Acknowledgements}

This work would never have been possible without the precious help of Borough Council President Sidi Othmane and the staff of his Hygiene Service. We would also like to thank the students of the Faculty of Sciences ben M'Sik for having contributed to the realization of the survey on the quality of life of diabetics.

\section{Conflicts of Interest}

The authors declare no conflicts of interest regarding the publication of this paper.

\section{References}

[1] International Diabetes Federation (2019) I. Atlas Diabetes. 9th Edition, Brussels. https://www.diabetesatlas.org

[2] World Health Organization (2016) Global Report on Diabetes. https://apps.who.int/iris/bitstream/handle/10665/204871/9789241565257 eng.pdf?s equence $=1$

[3] Whoqol, G. (1993) Study Protocol for the World Health Organization Project to Develop a Quality of Life Assessment Instrument (WHOQOL). Quality of Life Research, 2, 153-159. https://doi.org/10.1007/BF00435734 
[4] CDC (2016) Concept-Health Related Quality of Life. http://www.cdc.gov/hrqol/concept.htm

[5] Abouothman, S. (2014) Évaluation de la qualité de vie des patients diabétiques de type 2 au niveau de la région de Marrakech. University Cadi Ayyad, Faculty of Medicine and Pharmacy, Marrakech.

[6] Debout, C. (2011) The Concept of Quality of Life in Healthcare, a Complex Definition. Soins. La Revue de Reference Infirmiere, 56, 32-34.

[7] Fishwick, D., Lewis, L., Darby, A., Young, C., Wiggans, R., et al. (2015) Determinants of Health-Related Quality of Life among Residents with and without COPD in a Historically Industrialised Area. International Archives of Occupational and Environmental Health, 88, 799-805. https://doi.org/10.1007/s00420-014-1008-8

[8] Tan, Z., Liang, Y., Liu, S., Cao, W., Tu, H., et al. (2013) Health-Related Quality of Life as Measured with EQ-5D among Populations with and without Specific Chronic Conditions: A Population-Based Survey in Shaanxi Province, China. PLoS ONE, 8, e65958. https://doi.org/10.1371/journal.pone.0065958

[9] Liu, K., He, L., Tang, X., Wang, J., Li, N., et al. (2014) Relationship between Menopause and Health-Related Quality of Life in Middle-Aged Chinese Women: A CrossSectional Study. BMC Women's Health, 14, Article No. 7.

https://doi.org/10.1186/1472-6874-14-7

[10] Bryant, D., Schünemann, H., Brozek, J., Jaeschke, R. and Guyatt, G. (2007) Patient Reported Outcomes: General Principles of Development and Interpretability. Polskie Archiwum Medycyny Wewnetrznej, 117, 5-11. https://doi.org/10.20452/pamw.103

[11] Jackowski, D. and Guyatt, G. (2003) A Guide to Health Measurement. Clinical Orthopaedics and Related Research, 413, 80-89. https://doi.org/10.1097/01.blo.0000079771.06654.13

[12] Ware, J.E. and Sherbourne, C.D. (1992) The MOS 36-Item Short-Form Health Survey (SF-36). I. Conceptual Framework and Item Selection. Medical Care, 30, 473483. https://doi.org/10.1097/00005650-199206000-00002

[13] McHorney, C.A., Ware, J.E., Lu, J.F. and Sherbourne, C.D. (1994) The MOS 36Item Short-Form Health Survey (SF-36): III. Tests of Data Quality, Scaling Assumptions, and Reliability across Diverse Patient Groups. Medical Care, 32, 40-66. https://doi.org/10.1097/00005650-199401000-00004

[14] Ware, J., Kosinski, M. and Keller, S.D. (1996) A 12-Item Short-Form Health Survey: Construction of Scales and Preliminary Tests of Reliability and Validity. Medical Care, 34, 220-233. https://doi.org/10.1097/00005650-199603000-00003

[15] Brooks, R. (1996) EuroQol: The Current State of Play. Health Policy, 37, 53-72. https://doi.org/10.1016/0168-8510(96)00822-6

[16] Hunt, S.M., McKenna, S.P., McEwen, J., Williams, J., Papp, E. (1981) The Nottingham Health Profile: Subjective Health Status and Medical Consultations. Social Science \& Medicine, 15, 221-229. https://doi.org/10.1016/0271-7123(81)90005-5

[17] Bergner, M., Bobbitt, R.A., Carter, W.B. and Gilson, B.S. (1981) The Sickness Impact Profile: Development and Final Revision of a Health Status Measure. Medical Care, 19, 787-805. https://doi.org/10.1097/00005650-198108000-00001

[18] Rabin, R. and de Charro, F. (2001) EQ-5D: A Measure of Health Status from the EuroQol Group. Annals of Medicine, 33, 337-343. https://doi.org/10.3109/07853890109002087

[19] Euroqol Research Foundation (2018) EQ-5D-3L User Guide: Basic Information on 
How to Use EQ-5D-3L Instrument.

[20] Prieto, L. and Sacristán, J.A. (2004) What Is the Value of Social Values? The Uselessness of Assessing Health-Related Quality of Life through Preference Measures. BMC Medical Research Methodology, 4, Article No. 10.

https://doi.org/10.1186/1471-2288-4-10

[21] Cohen, J. (1988) Statistical Power Analysis for the Behavioral Sciences (2e édition). Lawrence Erlbaum Associates, Hillsdale.

[22] Lyhyaoui, O. (2011) Evaluation de la qualité de vie liée à la santé chez les diabétiques de type 2: Université Sidi Mohammed Ben Abdellah. Faculté de médecine et de pharmacie, Fès.

[23] Cardoso, A., Cruz, R., Queirós, P., Santiago, L., Ribeiro, C., et al. (2016) Assessment of Health-Related Quality of Life Using the EQ-5D-3L in Individuals with Type 2 Diabetes Mellitus. https://doi.org/10.15406/jdmdc.2016.03.00064

[24] Abedini, M.R., Bijari, B., Miri, Z., Shakhs Emampour, F. and Abbasi, A. (2020) The Quality of Life of the Patients with Diabetes Type 2 Using EQ-5D-5 L in Birjand. Health and Quality of Life Outcomes, 18, 18. https://doi.org/10.1186/s12955-020-1277-8

[25] McCaffrey, N., Kaambwa, B., Currow, D. and Ratcliffe, J. (2016) Health-Related Quality of Life Measured Using the EQ-5D-5L: South Australian Population Norms. Health and Quality of Life Outcomes, 14, 133. https://doi.org/10.1186/s12955-016-0537-0

[26] Tran, B.X., Ohinmaa, A., Nguyen, L.T., Nguyen, T.A. and Nguyen, T.H. (2011) Determinants of Health-Related Quality of Life in Adults Living with HIV in Vietnam. AIDS Care, 23, 1236-1245. https://doi.org/10.1080/09540121.2011.555749

[27] Quah, J.H., Luo, N., Ng, W.Y., How, C.H. and Tay, E.G. (2011) Health-Related Quality of Life Is Associated with Diabetic Complications, But Not with Short-Term Diabetic Control in Primary Care. ANNALS Academy of Medicine Singapore, 40, 276-286.

[28] Netuveli, G. and Blane, D. (2008) Quality of Life in Older Ages. British Medical Bulletin, 85, 113-126. https://doi.org/10.1093/bmb/ldn003

[29] O’Reilly, D.J., Xie, F., Pullenayegum, E., Gerstein, H.C., Greb, J., et al. (2011) Estimation of the Impact of Diabetes-Related Complications on Health Utilities for Patients with Type 2 Diabetes in Ontario, Canada. Quality of Life Research, 20, 939943. https://doi.org/10.1007/s11136-010-9828-9

[30] Altınok, A., Marakoğlu, K. and Kargın, N.Ç. (2016) Evaluation of Quality of Life and Depression Levels in Individuals with Type 2 Diabetes. Journal of Family Medicine and Primary Care, 5, 302-308. https://doi.org/10.4103/2249-4863.192358

[31] Bouffard, L. (2017) La Vie De Couple et Le Bonheur. Revue québécoise de psychologie, 38, 127-151. https://doi.org/10.7202/1040774ar

[32] Golicki, D. and Niewada, M. (2017) EQ-5D-5L Polish Population Norms. Archives of Medical Science: AMS, 13, 191-200. https://doi.org/10.5114/aoms.2015.52126

[33] Papazafiropoulou, A.K., Bakomitrou, F., Trikallinou, A., Ganotopoulou, A., Verras, C., et al. (2015) Diabetes-Dependent Quality of Life (ADDQOL) and Affecting Factors in Patients with Diabetes Mellitus Type 2 in Greece. BMC Research Notes, 8 , Article No. 786. https://doi.org/10.1186/s13104-015-1782-8

[34] Augustovski, F., Rey-Ares, L., Irazola, V., Garay, O.U., Gianneo, O., et al. (2016) An EQ-5D-5L Value Set Based on Uruguayan Population Preferences. Quality of Life Research, 25, 323-333. https://doi.org/10.1007/s11136-015-1086-4 
[35] Perneger, T.V., Combescure, C. and Courvoisier, D.S. (2010) General Population Reference Values for the French Version of the EuroQol EQ-5D Health Utility Instrument. Value Health, 13, 631-635.

https://doi.org/10.1111/j.1524-4733.2010.00727.x

[36] Sørensen, J., Davidsen, M., Gudex, C., Pedersen, K.M. and Brønnum-Hansen, H. (2009) Danish EQ-5D Population Norms. Scandinavian Journal of Public Health, 37, 467-474. https://doi.org/10.1177/1403494809105286

[37] Nguyen, L.H., Tran, B.X., Hoang Le, Q.N., Tran, T.T. and Latkin, C.A. (2017) Quality of Life Profile of General Vietnamese Population Using EQ-5D-5L. Health and Quality of Life Outcomes, 15, 199. https://doi.org/10.1186/s12955-017-0771-0

[38] Saleh, F., Ara, F., Mumu, S.J. and Hafez, M.A. (2015) Assessment of Health-Related Quality of Life of Bangladeshi Patients with Type 2 Diabetes Using the EQ-5D: A Cross-Sectional Study. BMC Research Notes, 8, Article No. 497.

https://doi.org/10.1186/s13104-015-1453-9

[39] Varghese, R.T., Salini, R., Abraham, P., Reeshma, K.K. and Vijayakumar, K. (2007) Determinants of the Quality of Life among Diabetic Subjects in Kerala, India. Diabetes \& Metabolic Syndrome: Clinical Research \& Reviews, 1, 173-179. https://doi.org/10.1016/j.dsx.2007.05.005

[40] Nyanzi, R., Wamala, R. and Atuhaire, L.K. (2014) Diabetes and Quality of Life: A Ugandan Perspective. Journal of Diabetes Research, 2014, Article ID: 402012. https://doi.org/10.1155/2014/402012

[41] Solli, O., Stavem, K. and Kristiansen, I.S. (2010) Health-Related Quality of Life in Diabetes: The Associations of Complications with EQ-5D Scores. Health and Quality of Life Outcomes, 8, Article No. 18. https://doi.org/10.1186/1477-7525-8-18

[42] American Diabetes Association (2020) 10. Cardiovascular Disease and Risk Management: Standards of Medical Care in Diabetes-2020. Diabetes Care, 43, S111S134. https://doi.org/10.2337/dc20-S010 